Original Research Article

\title{
Azidothymidine induces severe hematological toxicity and hepatic injury in Charles Foster rats
}

\author{
Rajat Pandey $^{1 *}$, Ramakant Singh ${ }^{2}$
}

${ }^{1}$ Department of Biotechnology, Vellore Institute of Technology University, Vellore, Tamilnadu, India

${ }^{2}$ Department of Toxicology, Central Drug Research Institute, Lucknow, Uttar Pradesh, India

Received: 20 July 2017

Revised: 25 July 2017

Accepted: 28 July 2017

*Correspondence to:

Dr. Rajat Pandey,

Email: pandey.rajat@

hotmail.com

Copyright: (C) the author(s), publisher and licensee Medip Academy. This is an openaccess article distributed under the terms of the Creative Commons Attribution NonCommercial License, which permits unrestricted noncommercial use, distribution, and reproduction in any medium, provided the original work is properly cited.

\begin{abstract}
Background: The present study aimed at evaluating the effects of azidothymidine (AZT) on hematologic and biochemical parameters in Charles Foster rats.

Methods: Twelve adult healthy Charles Foster rats comprising of six male and six females were selected for study. Test rodents were divided into four groups containing three rodents each. Three males and three females served as control and remaining received AZT drug. Rodents were acclimatized for 10 days and drug was administered for 28 days. After the completion of drug administration, blood samples were collected and analyzed for hematologic parameters, i.e., Hemoglobin (Hb), Packed cell volume (PCV), red blood cell (RBC), Mean corpuscular hemoglobin concentration (MCHC), total leukocyte count (TLC), Mean corpuscular volume (MCV), Platelet count (Plt) using a Fully Automatic Fully Digital Hematology Cell Counter. In addition, biochemical parameters, were measured to assess the effects of AZT on rodent physiology. In-vivo histopathological studies were also performed on vital organs of rodents to understand the effects of drug at tissue level.
\end{abstract}

Results: When compared with the control group, the data indicated a outstanding decrease in $\mathrm{Hb}, \mathrm{PCV}, \mathrm{RBC}$, TLC and platelets in all test groups, whereas MCHC did not show any major reduction but MCV data suggested a slight increase. Among biochemical parameters, aspartate aminotransferase (AST), alanine aminotransferase (ALT) and alkaline phosphatase (ALP). were found to be remarkably elevated along with elevated bilirubin and reduced albumin, pointing towards a possible liver damage which was later corroborated by liver histopathological study.

Conclusions: Above results indicate azidothymidine to be a myelosuppresive and hepatotoxic drug and its usage as an anti-retro viral during highly active antiretro viral therapy (HAART) regime should be strictly monitored.

Keywords: AZT, Hematotoxicity, Hepatotoxicity, Subacute toxicity

\section{INTRODUCTION}

Human immunodeficiency virus infection and acquired immune deficiency syndrome (HIV/AIDS) is a spectrum of conditions caused by the human immunodeficiency virus and it remains one of the world's most significant public health challenges, particularly in low and middleincome countries. ${ }^{1}$ As per a WHO report, in 2015 about 36.7 million people were living with HIV and it resulted in 1.2 million deaths Most of those infected live in subSaharan Africa. ${ }^{2}$ According to a statistical report of CDC (centre for disease control) between its discovery and 2014 AIDS has caused an estimated 39 million deaths worldwide. $^{3}$

AIDS is a lethal multi-system disease that has become a major public health problem since its recognition in 1981. ${ }^{4,5}$ The etiological agent of AIDS is a retrovirus referred to as HIV. ${ }^{6}$ There is no certain cure or vaccine for the treatment of HIV; treatment consists of highly active antiretroviral therapy (HAART) which slows progression of the disease. ${ }^{7}$ Current HAART options are combinations 
(or "cocktails") consisting of at least three medications belonging to at least two types, or "classes," of antiretroviral agents. HIV medicines are grouped into six drug classes according to how they fight HIV.

The six drug classes are:

1. Non-nucleoside reverse transcriptase inhibitors (NNRTIs),

2. Nucleoside reverse transcriptase inhibitors (NRTIs),

3. Protease inhibitors (PIs),

4. Fusion inhibitors,

5. CCR5 antagonists (CCR5s),

6. Integrase strand transfer inhibitors (INSTIs)

These six drug classes include more than 25 HIV medicines that are approved to treat HIV infection. Azidothymidine commonly referred to as AZT was the first drug to be approved by FDA for AIDS treatment. ${ }^{8-10}$ AZT is a synthetic analogue of thymidine (3'-azido-3'deoxythymidine) and a reverse transcriptase inhibitor used in combination with other agents in the therapy and prophylaxis of the human immunodeficiency virus (HIV) infection and the acquired immunodeficiency syndrome (AIDS). ${ }^{11}$ AZT is phosphorylated intracellularly and then acts in competing with the natural substrate, thymidine triphosphate, for incorporation into growing HIV DNA chain causing inhibition of the viral reverse transcriptase and chain termination. Recently, AZT has been replaced by better tolerated nucleoside analogues and it is no longer commonly used in developed countries but that's not the case with developing or under developed countries because of the drug's affordability. ${ }^{12}$

The most adverse effect of AZT are anaemia and granulocytopenia, which are believed to reflect bone marrow toxicity. ${ }^{8,13}$ Two types of anaemia may occur with AZT therapy marcrocytic megaloblastic anaemia and normochromic anaemia. ${ }^{14,15}$ Several subacute and subchronic rodent toxicity studies have demonstrated that the primary toxicity of AZT is myelosuppression. A recent study evaluated the effects of AZT on hemostatic and hematologic parameters in Wistar rats and found out a significant decrease in RBCs, PCV and platelet count. ${ }^{16}$ In another study it was demonstrated that AZT treatment can cause hepatotoxicity in Wistar rats. ${ }^{17}$ They reported highly significant increase in alanine transaminase, alkaline phosphatase, argininosuccinic acid lyase and bilirubin in serum of Wistar albino rats. In a similar kind of study it was reported that AZT can cause significant increase in serum levels of aspartate aminotransferase (AST), alanine aminotransferase (ALT) and alkaline phosphatase (ALP). ${ }^{18}$ Apart from rodent models, AZT has also shown to cause severe clinical problems in humans as well. A group of researchers reported AZT associated toxicity in HIV infected patients, they reported severe anaemia in significantly higher number of patients, moreover female patients were reported to be more prone to anaemia as compared to male patients. ${ }^{19}$ In the present study, we have investigated the effects of an anti-retro viral drug
Azidothymidine on hematological parameters of Charles foster rats. We have also studied the hepatotoxic effects of Azidothymidine on the liver of Charles Foster rats by histopathological studies and quantifying important serum marker enzymes along with bilirubin and albumin.

\section{METHODS}

The drug which was tested for hematotoxicity in the present study was Azidothymidine (AZT) Brand name: Zidovir (Cipla pharmaceuticals) is available in $300 \mathrm{mg}$ Tablet form and was purchased from local pharmacy shop in Lucknow, India.

\section{In vivo cytotoxicity studies}

\section{Experimental rodents}

6 Male and 6 Female Charles foster strains of Rats, weight ranging from 200 to $300 \mathrm{~g}$ were used. These rodents were supplied for the experiment, by the Division of Laboratory animals, CDRI, Lucknow. Proper approvals were obtained from ethical committee of CDRI, Lucknow before starting of this work. Rats were quarantined for 10 days and it was confirmed that they were free of pathogen (Ecto and Endo Parasites- Mycoplasma, Pasteurella, Rat pneumenitis virus etc.) After acclimatization, 12 healthy young and active albino rats of charles foster strain comprising of 6 male and 6 female rats were selected based on their body weight and by assessing initial hematological parameters by collecting $1 \mathrm{ml}$ fresh blood sample in $0.1 \%$ EDTA collection vials. The rodents which looked sick or those that had initial hematological parametric values out of the range were discarded. The female rats were nulliparous and nonpregnant. Rats were divided in to two groups of 6 rats having 3 males and 3 females, and were kept under conventional condition (open system) and housed in plastic cages (floor area $800 \mathrm{~cm}^{2}$, height $14 \mathrm{~cm}$.) on sterilized rice bran bedding. They were provided with standard rodent pellets diet procured from Ashirvad Ltd. and filter water from Aqua guard in $300 \mathrm{ml}$ bottles ( $24 \mathrm{hrs}$ ). Rats were housed in a room where room temperature was kept at 22 degree Celsius, relative humidity was controlled between $50-70 \%$ and 12-hour light and 12-hour dark photo period was provided. The first group served as control group (Group I) and second as treated (Group II).

\section{Dose}

The dose selected was 180mg AZT per kilogram body weight. The drug was mixed with appropriate amount of water and grinded in mortar and pestle (glass). The fine drug solution was then given to the rats of treated group (oral route) with the help of cannula.

\section{In-vivo hematological studies}

Important hematological parameters were assessed at the end of the experiment i.e. 28 days of dosing using MS9 Fully Automatic Fully Digital Hematology Cell Counter. 
Version $3.5 \times \mathrm{E}$ from Melet Schloesing Laboratories, France. Hemoglobin, Hematocrit (PCV), T-RBC, MCHC, TLC, MCV and Platelet were the hematological parameters studied.

\section{Biochemical analysis}

The cardiovascular blood was removed after sacrificing the rodents. The Blood was allowed to clot and was subjected to centrifugation at $3000 \mathrm{rpm}$ for 10 minutes. The serum obtained was further analyzed in BECKMANN Synchron clinical system CX4/CX5, made in USA for the following parameters: Glucose, Cholesterol, Triglycerides, Total Protein, Albumin, Alkaline aminotransferase, Aspartate aminotransferase, Alkaline Phosphatase, Total Bilirubin, Blood Urea Nitrogen, Creatinine, Calcium and Phosphate.

\section{Dissection}

The rats were anesthetized by anesthetic ether and then were dissected. The cardiovascular blood was collected and allowed to clot for serum isolation and further biochemical analysis. The different organs removed and further processed were: Brain, Trachea, Lung, Heart, liver, spleen, adrenal gland, kidney, reproductive system. Absolute and relative organ weights were recorded.

\section{In vivo histopathological studies}

Systemic studies of the Drug were analyzed by standard Histopathological techniques including Dissection, Tissue processing and Slide preparation.

\section{RESULTS}

\section{Hematotoxicological manifestations of AZT}

Table 1 and Table 2 shows the data of hematological parameters of AZT treated as well as control rats. It was observed that following AZT administration for 28 days, the mean values of Hemoglobin, packed cell volume, $\mathrm{RBC}, \mathrm{WBC}$ and platelets remarkably reduced in male as well as female treated rats, when compared to control.

Table 1: Recording of hematological parameters of AZT treated rats.

\begin{tabular}{|c|c|c|c|c|c|c|c|c|c|c|c|c|c|c|}
\hline & \multicolumn{2}{|c|}{ Hb (g/dl) } & \multicolumn{2}{|c|}{ PCV $(\%)$} & \multicolumn{2}{|c|}{$\begin{array}{l}\mathrm{RBC} \\
\left(10^{6} \text { cells/ml }\right)\end{array}$} & \multicolumn{2}{|c|}{ MCHC (g/dl) } & \multicolumn{2}{|c|}{$\begin{array}{l}\text { TLC }\left(10^{3}\right. \\
\text { cells } / \mathrm{ml})\end{array}$} & \multicolumn{2}{|c|}{$\operatorname{MCV}(\mu)$} & \multicolumn{2}{|c|}{$\begin{array}{l}\text { PIt }\left(10^{3}\right. \\
\text { cells } / \mathrm{ml})\end{array}$} \\
\hline & $\begin{array}{l}1^{\text {st }} \\
\text { day }\end{array}$ & $\begin{array}{l}28^{\text {th }} \\
\text { day }\end{array}$ & $\begin{array}{l}1^{\text {st }} \\
\text { day }\end{array}$ & $\begin{array}{l}28^{\text {th }} \\
\text { day }\end{array}$ & $\begin{array}{l}1^{\text {st }} \\
\text { day }\end{array}$ & $\begin{array}{l}28^{\text {th }} \\
\text { day }\end{array}$ & $\begin{array}{l}1^{\text {st }} \\
\text { day }\end{array}$ & $\begin{array}{l}28^{\text {th }} \\
\text { day }\end{array}$ & $\begin{array}{l}1^{\text {st }} \\
\text { day }\end{array}$ & $\begin{array}{l}28^{\text {th }} \\
\text { day }\end{array}$ & $\begin{array}{l}1^{\text {st }} \\
\text { day }\end{array}$ & $\begin{array}{l}28^{\text {th }} \\
\text { day }\end{array}$ & $\begin{array}{l}1^{\text {st }} \\
\text { day }\end{array}$ & $\begin{array}{l}28^{\text {th }} \\
\text { day }\end{array}$ \\
\hline $1 \mathrm{~F}$ & $\begin{array}{l}12.8 \pm \\
1.8\end{array}$ & $\begin{array}{l}4.5 \pm \\
0.5\end{array}$ & $\begin{array}{l}30.8 \pm \\
5.6\end{array}$ & $\begin{array}{l}12.9 \pm \\
1.8\end{array}$ & $\begin{array}{l}4.5 \pm \\
0.9\end{array}$ & $\begin{array}{l}1.8 \pm \\
0.3\end{array}$ & $\begin{array}{l}41.6 \pm \\
6.4\end{array}$ & $\begin{array}{l}39.8 \pm \\
4.1\end{array}$ & $\begin{array}{l}11.5 \pm \\
1.6\end{array}$ & $\begin{array}{l}4.6 \pm \\
0.9\end{array}$ & $\begin{array}{l}69.2 \pm \\
6.9\end{array}$ & $\begin{array}{l}72.2 \pm \\
5.2\end{array}$ & $\begin{array}{l}384 \pm \\
23\end{array}$ & $\begin{array}{l}143 \pm \\
12\end{array}$ \\
\hline $2 \mathrm{~F}$ & $\begin{array}{l}13.7 \pm \\
1.7\end{array}$ & $\begin{array}{l}5.6 \pm \\
0.9\end{array}$ & $\begin{array}{l}36.1 \pm \\
5.2\end{array}$ & $\begin{array}{l}12.9 \pm \\
3.2\end{array}$ & $\begin{array}{l}5.8 \pm \\
1.0\end{array}$ & $\begin{array}{l}2.3 \pm \\
0.2\end{array}$ & $\begin{array}{l}38.0 \pm \\
6.2\end{array}$ & $\begin{array}{l}42.5 \pm \\
3.7\end{array}$ & $\begin{array}{l}9.9 \pm \\
1.0\end{array}$ & $\begin{array}{l}3.2 \pm \\
0.6\end{array}$ & $\begin{array}{l}62.2 \pm \\
7.1\end{array}$ & $\begin{array}{l}67.3 \pm \\
4.1\end{array}$ & $\begin{array}{l}315 \pm \\
34\end{array}$ & $\begin{array}{l}108 \pm \\
11\end{array}$ \\
\hline $3 \mathrm{~F}$ & $\begin{array}{l}12.5 \pm \\
2.1\end{array}$ & $\begin{array}{l}5.5 \pm \\
1.1\end{array}$ & $\begin{array}{l}35.4 \pm \\
6.3\end{array}$ & $\begin{array}{l}15.6 \pm \\
3.8\end{array}$ & $\begin{array}{l}5.9 \pm \\
1.2\end{array}$ & $\begin{array}{l}1.9 \pm \\
0.4\end{array}$ & $\begin{array}{l}35.4 \pm \\
3.0\end{array}$ & $\begin{array}{l}38.1 \pm \\
3.0\end{array}$ & $\begin{array}{l}12.8 \pm \\
1.8\end{array}$ & $\begin{array}{l}3.5 \pm \\
0.5\end{array}$ & $\begin{array}{l}60.0 \pm \\
4.5\end{array}$ & $\begin{array}{l}65.3 \pm \\
4.9\end{array}$ & $\begin{array}{l}272 \pm \\
28\end{array}$ & $\begin{array}{l}155 \pm \\
16\end{array}$ \\
\hline $7 \mathrm{M}$ & $\begin{array}{l}15.8 \pm \\
2.2\end{array}$ & $\begin{array}{l}6.1 \pm \\
0.8\end{array}$ & $\begin{array}{l}43.1 \pm \\
7.8\end{array}$ & $\begin{array}{l}14.9 \pm \\
2.9\end{array}$ & $\begin{array}{l}6.1 \pm \\
1.2 \\
\end{array}$ & $\begin{array}{l}1.9 \pm \\
0.4\end{array}$ & $\begin{array}{l}36.8 \pm \\
4.1\end{array}$ & $\begin{array}{l}37.1 \pm \\
3.9\end{array}$ & $\begin{array}{l}10.3 \pm \\
2.1\end{array}$ & $\begin{array}{l}4.9 \pm \\
0.6\end{array}$ & $\begin{array}{l}63.2 \pm \\
5.9\end{array}$ & $\begin{array}{l}68.9 \pm \\
5.3\end{array}$ & $\begin{array}{l}328 \pm \\
31\end{array}$ & $\begin{array}{l}135 \pm \\
15\end{array}$ \\
\hline $8 \mathrm{M}$ & $\begin{array}{l}15.7 \pm \\
1.6\end{array}$ & $\begin{array}{l}6.2 \pm \\
0.6\end{array}$ & $\begin{array}{l}40.5 \pm \\
6.9\end{array}$ & $\begin{array}{l}19.7 \pm \\
4.4\end{array}$ & $\begin{array}{l}6.2 \pm \\
1.3\end{array}$ & $\begin{array}{l}1.8 \pm \\
0.3\end{array}$ & $\begin{array}{l}38.8 \pm \\
3.4\end{array}$ & $\begin{array}{l}37.8 \pm \\
4.2\end{array}$ & $\begin{array}{l}11.6 \pm \\
1.5\end{array}$ & $\begin{array}{l}5.8 \pm \\
0.8\end{array}$ & $\begin{array}{l}64.9 \pm \\
5.1\end{array}$ & $\begin{array}{l}67.5 \pm \\
4.5\end{array}$ & $\begin{array}{l}419 \pm \\
39\end{array}$ & $\begin{array}{l}238 \pm \\
32\end{array}$ \\
\hline $9 \mathrm{M}$ & $\begin{array}{l}14.9 \pm \\
2.3\end{array}$ & $\begin{array}{l}7.4 \pm \\
0.9\end{array}$ & $\begin{array}{l}39.1 \pm \\
4.3\end{array}$ & $\begin{array}{l}17.2 \pm \\
3.2\end{array}$ & $\begin{array}{l}6.1 \pm \\
1.3\end{array}$ & $\begin{array}{l}2.3 \pm \\
0.2\end{array}$ & $\begin{array}{l}38.2 \pm \\
5.4\end{array}$ & $\begin{array}{l}36.5 \pm \\
5.1\end{array}$ & $\begin{array}{l}9.4 \pm \\
1.3\end{array}$ & $\begin{array}{l}4.3 \pm \\
0.7\end{array}$ & $\begin{array}{l}63.8 \pm \\
6.1\end{array}$ & $\begin{array}{l}70.2 \pm \\
4.9\end{array}$ & $\begin{array}{l}343 \pm \\
22\end{array}$ & $\begin{array}{l}125 \pm \\
18\end{array}$ \\
\hline
\end{tabular}

Table 2: Recording of hematological parameters of control rats.

\begin{tabular}{|c|c|c|c|c|c|c|c|c|c|c|c|c|c|c|}
\hline & \multicolumn{2}{|c|}{ Hb (g/dl) } & \multicolumn{2}{|c|}{ PCV (\%) } & \multicolumn{2}{|c|}{$\begin{array}{l}\text { RBC } \\
\left(10^{6} \text { cells } / \mathrm{ml}\right)\end{array}$} & \multicolumn{2}{|c|}{ MCHC (g/dl) } & \multicolumn{2}{|c|}{$\begin{array}{l}\mathrm{TLC}\left(\mathbf{1 0}^{\mathbf{3}}\right. \\
\text { cells/ml) }\end{array}$} & \multicolumn{2}{|c|}{$\operatorname{MCV}(\mu)$} & \multicolumn{2}{|c|}{$\begin{array}{l}\text { Plt }\left(10^{3}\right. \\
\text { cells } / \mathbf{m l})\end{array}$} \\
\hline & $\begin{array}{l}1^{\text {st }} \\
\text { day }\end{array}$ & $\begin{array}{l}28^{\text {th }} \\
\text { day }\end{array}$ & $\begin{array}{l}1^{\text {st }} \\
\text { day }\end{array}$ & $\begin{array}{l}28^{\text {th }} \\
\text { day }\end{array}$ & $\begin{array}{l}1^{\text {st }} \\
\text { day }\end{array}$ & $\begin{array}{l}28^{\text {th }} \\
\text { day }\end{array}$ & $\begin{array}{l}1^{\text {st }} \\
\text { day }\end{array}$ & $\begin{array}{l}28^{\text {th }} \\
\text { day }\end{array}$ & $\begin{array}{l}1^{\text {st }} \\
\text { day }\end{array}$ & $\begin{array}{l}28^{\text {th }} \\
\text { day }\end{array}$ & $\begin{array}{l}1^{\text {st }} \\
\text { day }\end{array}$ & $\begin{array}{l}28^{\text {th }} \\
\text { day }\end{array}$ & $\begin{array}{l}1^{\text {st }} \\
\text { day }\end{array}$ & $\begin{array}{l}28^{\text {th }} \\
\text { day }\end{array}$ \\
\hline $4 \mathrm{~F}$ & $\begin{array}{l}14.4 \pm \\
1.4\end{array}$ & $\begin{array}{l}14.3 \pm \\
1.3\end{array}$ & $\begin{array}{l}33.1 \pm \\
3.2\end{array}$ & $\begin{array}{l}39.7 \pm \\
4.2\end{array}$ & $\begin{array}{l}4.7 \pm \\
0.8\end{array}$ & $\begin{array}{l}4.9 \pm \\
0.6\end{array}$ & $\begin{array}{l}43.5 \pm \\
2.9\end{array}$ & $\begin{array}{l}42.3 \pm \\
3.7\end{array}$ & $\begin{array}{l}7.8 \pm 0 . \\
6\end{array}$ & $\begin{array}{l}8.3 \pm \\
0.7\end{array}$ & $\begin{array}{l}70.0 \pm \\
6.3\end{array}$ & $\begin{array}{l}68.5 \pm \\
5.1\end{array}$ & $\begin{array}{l}176 \pm \\
22\end{array}$ & $\begin{array}{l}173 \pm \\
24\end{array}$ \\
\hline $5 \mathrm{~F}$ & $\begin{array}{l}13.9 \pm \\
1.1\end{array}$ & $\begin{array}{l}14.5 \pm \\
1.5\end{array}$ & $\begin{array}{l}37.1 \pm \\
3.3\end{array}$ & $\begin{array}{l}38.2 \pm \\
4.0\end{array}$ & $\begin{array}{l}5.6 \pm \\
0.9\end{array}$ & $\begin{array}{l}5.8 \pm \\
0.9\end{array}$ & $\begin{array}{l}37.3 \pm \\
3.5\end{array}$ & $\begin{array}{l}38.0 \pm \\
3.0\end{array}$ & $\begin{array}{l}11.2 \pm \\
0.9\end{array}$ & $\begin{array}{l}10.4 \\
\pm 0.8\end{array}$ & $\begin{array}{l}66.0 \pm \\
4.3 \\
\end{array}$ & $\begin{array}{l}66.3 \pm \\
5.4\end{array}$ & $\begin{array}{l}348 \pm \\
30\end{array}$ & $\begin{array}{l}494 \pm \\
44\end{array}$ \\
\hline $6 \mathrm{~F}$ & $\begin{array}{l}13.3 \pm \\
1.5\end{array}$ & $\begin{array}{l}12.9 \pm \\
1.1\end{array}$ & $\begin{array}{l}37.5 \pm \\
2.9\end{array}$ & $\begin{array}{l}34.3 \pm \\
3.7\end{array}$ & $\begin{array}{l}5.9 \pm \\
0.7\end{array}$ & $\begin{array}{l}5.3 \pm \\
0.7\end{array}$ & $\begin{array}{l}40.8 \pm \\
3.1\end{array}$ & $\begin{array}{l}37.5 \pm \\
3.9\end{array}$ & $\begin{array}{l}10.4 \pm \\
1.1\end{array}$ & $\begin{array}{l}9.7 \pm \\
0.7\end{array}$ & $\begin{array}{l}63.3 \pm \\
4.8\end{array}$ & $\begin{array}{l}61.0 \pm \\
4.4\end{array}$ & $\begin{array}{l}311 \pm \\
45\end{array}$ & $\begin{array}{l}289 \pm \\
23\end{array}$ \\
\hline $10 \mathrm{M}$ & $\begin{array}{l}15.7 \pm \\
0.9\end{array}$ & $\begin{array}{l}15.7 \pm \\
1.8\end{array}$ & $\begin{array}{l}43.0 \pm \\
5.6\end{array}$ & $\begin{array}{l}41.9 \pm \\
3.0\end{array}$ & $\begin{array}{l}6.7 \pm \\
1.2\end{array}$ & $\begin{array}{l}6.2 \pm \\
0.5\end{array}$ & $\begin{array}{l}36.5 \pm \\
3.2\end{array}$ & $\begin{array}{l}37.6 \pm \\
3.1\end{array}$ & $\begin{array}{l}10.4 \pm \\
1.0\end{array}$ & $\begin{array}{l}15.1 \\
\pm 1.1\end{array}$ & $\begin{array}{l}64.1 \pm \\
5.3\end{array}$ & $\begin{array}{l}62.3 \pm \\
3.3\end{array}$ & $\begin{array}{l}304 \pm \\
27\end{array}$ & $\begin{array}{l}641 \pm \\
57\end{array}$ \\
\hline $11 \mathrm{M}$ & $\begin{array}{l}13.3 \pm \\
1.3\end{array}$ & $\begin{array}{l}13.9 \pm \\
1.4\end{array}$ & $\begin{array}{l}36.6 \pm \\
3.9\end{array}$ & $\begin{array}{l}36.6 \pm \\
4.2\end{array}$ & $\begin{array}{l}6.1 \pm \\
1.0\end{array}$ & $\begin{array}{l}5.8 \pm \\
0.7\end{array}$ & $\begin{array}{l}36.4 \pm \\
3.3\end{array}$ & $\begin{array}{l}38.0 \pm \\
3.0\end{array}$ & $\begin{array}{l}9.9 \pm 0 . \\
8\end{array}$ & $\begin{array}{l}11.3 \\
\pm 0.8\end{array}$ & $\begin{array}{l}59.8 \pm \\
4.9\end{array}$ & $\begin{array}{l}58.6 \pm \\
4.9\end{array}$ & $\begin{array}{l}221 \pm \\
19\end{array}$ & $\begin{array}{l}278 \pm \\
26\end{array}$ \\
\hline $12 \mathrm{M}$ & $\begin{array}{l}11.6 \pm \\
1.2\end{array}$ & $\begin{array}{l}16.1 \pm \\
1.6\end{array}$ & $\begin{array}{l}31.3 \pm \\
4.1\end{array}$ & $\begin{array}{l}44.6 \pm \\
4.4\end{array}$ & $\begin{array}{l}4.5 \pm \\
0.8\end{array}$ & $\begin{array}{l}6.2 \pm \\
0.9\end{array}$ & $\begin{array}{l}37.2 \pm \\
3.3\end{array}$ & $\begin{array}{l}36.1 \pm \\
4.2\end{array}$ & $\begin{array}{l}7.8 \pm 0 . \\
9\end{array}$ & $\begin{array}{l}12.1 \\
\pm 1.0\end{array}$ & $\begin{array}{l}69.2 \pm \\
5.4\end{array}$ & $\begin{array}{l}70.5 \pm \\
5.0\end{array}$ & $\begin{array}{l}280 \pm \\
33\end{array}$ & $\begin{array}{l}586 \pm \\
41\end{array}$ \\
\hline
\end{tabular}




\section{Serum marker enzymes and bilirubin}

The standard serum enzymes predicting hepatotoxicity and their respective activities are presented in Table 3 and Table 4. Results suggest a notable elevation in the levels of all three serum marker enzymes i.e. ALT, ASP, ALP along with total bilirubin content in AZT treated rats. Male as well as female rats showed the similar pattern of elevation as compared to control rats.

Table 3: Recording of biochemical parameters of AZT treated rats.

\begin{tabular}{|llllll|}
\hline & $\begin{array}{l}\text { ALB } \\
(\mathrm{g} / \mathrm{dl})\end{array}$ & $\begin{array}{l}\text { ALT } \\
(\text { IU/l) }\end{array}$ & $\begin{array}{l}\text { AST } \\
(\text { IU/l) }\end{array}$ & $\begin{array}{l}\text { ALP } \\
(\text { IU/l) }\end{array}$ & $\begin{array}{l}\text { TBIL } \\
(\mathrm{mg} / \mathrm{dl})\end{array}$ \\
\hline $1 \mathrm{~F}$ & $1.6 \pm 0.2$ & $75 \pm 4.1$ & $301 \pm 23$ & $224 \pm 14$ & $0.6 \pm 0.09$ \\
\hline $2 \mathrm{~F}$ & $2.0 \pm 0.1$ & $70 \pm 3.3$ & $283 \pm 21$ & $216 \pm 8$ & $0.7 \pm 0.1$ \\
\hline $3 \mathrm{~F}$ & $1.3 \pm 0.1$ & $66 \pm 2.9$ & $288 \pm 26$ & $264 \pm 12$ & $0.5 \pm 0.1$ \\
\hline $7 \mathrm{M}$ & $1.4 \pm 0.2$ & $72 \pm 3.6$ & $367 \pm 31$ & $236 \pm 16$ & $0.6 \pm 0.08$ \\
\hline $8 \mathrm{M}$ & $1.7 \pm 0.1$ & $69 \pm 3.9$ & $292 \pm 29$ & $250 \pm 7$ & $0.6 \pm 0.1$ \\
\hline $9 \mathrm{M}$ & $1.7 \pm 0.2$ & $73 \pm 3.2$ & $350 \pm 21$ & $230 \pm 11$ & $0.8 \pm 0.2$ \\
\hline
\end{tabular}

ALB: Albumin; ALT: Alanine aminotransferase; AST: Aspartate aminotransferase;

ALP: Alkaline phosphatase; TBIL: Total bilirubin

Table 4: Recording of biochemical parameters of control rats.

\begin{tabular}{|llllll||}
\hline & $\begin{array}{l}\text { ALB } \\
(\mathrm{g} / \mathrm{dl})\end{array}$ & $\begin{array}{l}\text { ALT } \\
(\text { IU/l) }\end{array}$ & $\begin{array}{l}\text { AST } \\
(\text { IU/l) }\end{array}$ & $\begin{array}{l}\text { ALP } \\
(\text { IU/l) }\end{array}$ & $\begin{array}{l}\text { TBIL } \\
(\mathrm{mg} / \mathrm{dl})\end{array}$ \\
\hline $4 \mathrm{~F}$ & $3.6 \pm 0.2$ & $38 \pm 1.8$ & $160 \pm 14$ & $95 \pm 6$ & $0.1 \pm 0.0$ \\
\hline $5 \mathrm{~F}$ & $3.8 \pm 0.4$ & $42 \pm 2.1$ & $166 \pm 11$ & $114 \pm 10$ & $0.2 \pm 0.0$ \\
\hline $6 \mathrm{~F}$ & $4.0 \pm 0.3$ & $40 \pm 3.4$ & $159 \pm 13$ & $120 \pm 12$ & $0.15 \pm 0.0$ \\
\hline $10 \mathrm{M}$ & $4.1 \pm 0.2$ & $41 \pm 3.1$ & $171 \pm 12$ & $114 \pm 9$ & $0.12 \pm 0.01$ \\
\hline $11 \mathrm{M}$ & $4.3 \pm 0.3$ & $42 \pm 2.7$ & $161 \pm 9$ & $134 \pm 11$ & $0.22 \pm 0.05$ \\
\hline $12 \mathrm{M}$ & $4.8 \pm 0.1$ & $44 \pm 4.2$ & $160 \pm 15$ & $131 \pm 13$ & $0.25 \pm 0.03$ \\
\hline
\end{tabular}

ALB: Albumin; ALT: Alanine aminotransferase; AST: Aspartate aminotransferase;

ALP: Alkaline phosphatase; TBIL: Total bilirubin

\section{Effect of AZT on histopathology of liver}

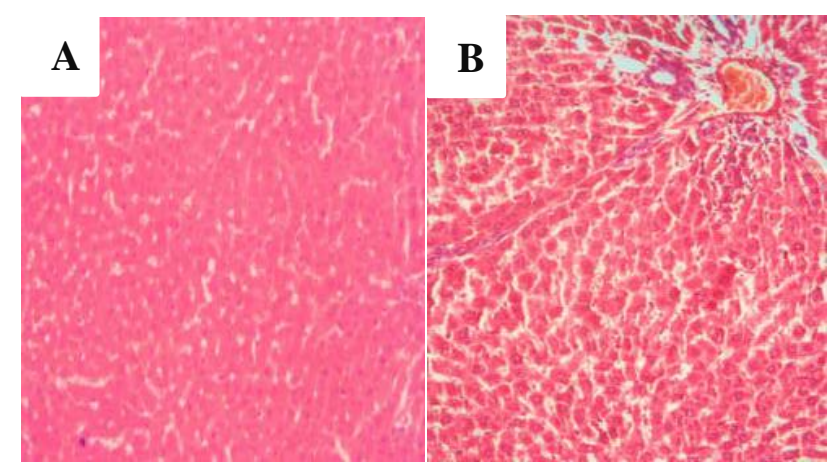

Figure 1: A) Control rat, B) AZT treated rat; Histopathology of Charles Foster rat liver.

The histopathological effects of AZT on the liver of Charles foster rat is presented in Figure 1. The liver of treated rat is showing inflammatory cells around the central vein owing to AZT toxicity, the image also shows an enlargement of the central vein of the liver. The liver is highly susceptible to xenobiotic-induced injury because of its central role in foreign chemical substance metabolism.

\section{DISCUSSION}

The findings suggest AZT to have an inhibitory effect on hemopoiesis as reported previously. ${ }^{16}$ AZT therapy is probably the most common cause of anaemia in HIVinfected patients. In the clinical trial studies of AZT in patients with advanced stage of HIV, statistically significant reductions in hemoglobin levels occurred in $34 \%$ of subjects receiving AZT $(1,200 \mathrm{mg}$ per day) following 6 weeks of therapy. ${ }^{13}$ Our data suggests a drastic reduction in haemoglobin level of AZT treated rats as compared to control. A similar kind of study reported an increase in erythrocyte mean corpuscular volume (MCV) which finds agreement with our data where AZT treated male and female rats have slightly increased MCV as compared to control rodents. ${ }^{13}$ Apart from anaemia, the most common effect observed in-vivo during AZT treatment is neutropenia, which seems to have direct suppressive effect on heme synthesis. ${ }^{20}$ The high dose of heme analogue compounds which are used for treatment of heavy metal induced hematotoxicity, may cause anaemia because of their modulatory effects on the enzymes involved in heme synthesis. ${ }^{21}$ Some studies report increased WBC counts, following AZT administration while some authors report otherwise. ${ }^{16,22-24}$ Increased WBC count maybe attributed to inflammation due to chronic infection, but in the present study WBC count was reduced post drug administration. Our data suggests that AZT may have a leucopenic inhibitory effect in Charles foster rat. Our study also indicated a reduction in the platelet count as reported previously. ${ }^{16,23}$ A previous study also reported platelet degradation upon high dose of AZT administration. ${ }^{25}$

Preceding literature suggests AZT induced elevation in the levels of transaminases and ALP in human and albino rats has already been reported. ${ }^{18,26}$ The data presented in this report indicates towards a hepatic injury in AZT treated Charles Foster rats. Elevated bilirubin levels were reported in humans and rats upon AZT administration, and our results are in agreement with these reports. ${ }^{18,27}$ Low titers of serum albumin in AZT treated rats also point towards a possible liver injury. Albumin is produced by hepatocytes and hence determining serum albumin levels is often considered "tests of liver function." This is mainly because hepatic synthesis of albumin tends to decrease in end-stage liver disease.

Liver occupies a portal location within the circulatory system. ${ }^{28}$ Hepatotoxicity is the most adverse reaction of AZT treatment but still there is no clear explanation of its mechanism mainly because manifestations of AZT induced hepatotoxicity are like other conditions of hepatic injuries but it is very much evident from the images that 
AZT caused an abnormal morphology of the liver. ${ }^{17,29}$ Transaminases and ALP are considered important markers of hepatic injury because their increased level in blood serum is observed immediately post hepatic necrosis and hepatocellular membrane damage. ${ }^{27,30}$ In the present study, AZT treated rodents showed a highly remarkable increase in ALT and ALP and these results are in agreement with the above report. In the present study, AZT treated rodents showed increased bilirubin levels and this observation demonstrate that this toxin-induced insult to the liver could also result in hyperbilirubinemia in addition to hepatocellular necrosis.

Funding: No funding sources

Conflict of interest: None declared

Ethical approval: The study was approved by the Institutional Ethics Committee of CDRI, Lucknow

\section{REFERENCES}

1. Sepkowitz KA. AIDS--the first 20 years. N Engl J Med. 2001;344(23):1764-1772.

2. WHO | HIV/AIDS. WHO. Available at: http://www.who.int/mediacentre/factsheets/fs360/en/. Accessed May 1, 2017.

3. HIV Basics CDC. Available at: https://www.cdc.gov/hiv/basics/statistics.html. Accessed May 1, 2017.

4. Gottlieb MS, Schroff R, Schanker HM, Weisman JD, Fan PT, Wolf RA, et al. Pneumocystis carinii pneumonia and mucosal candidiasis in previously healthy homosexual men: evidence of a new acquired cellular immunodeficiency. New England Journal of Medicine. 1981 Dec 10;305(24):1425-31.

5. Masur H, Michelis MA, Greene JB. An outbreak of community-acquired Pneumocystis carinii pneumonia: initial manifestation of cellular immune dysfunction. N Engl J Med. 1981;305(24):1431-8.

6. Coffin J, Haase A, Levy JA, Montagnier L, Oroszlan S, Teich N, Temin H, Toyoshima K, Varmus H, Vogt P. What to call the AIDS virus?. Nature. 1986;321(6065):10.

7. Rerks-Ngarm S, Paris RM, Chunsutthiwat S, Premsri N, Namwat C, Bowonwatanuwong C, et al. Extended evaluation of the virologic, immunologic, and clinical course of volunteers who acquired HIV-1 infection in a phase III vaccine trial of ALVAC-HIV and AIDSVAX B/E. The Journal of infectious diseases. 2012 Jul 26;207(8):1195-205.

8. Amin NM. Zidovudine for treating AIDS. What physicians need to know. Postgrad Med. 1989;86(1):195-6,201-8.

9. Vince R, Hua M, Brownell J, Daluge S, Lee F, Shannon WM, et al. Potent and selective activity of a new carbocyclic nucleoside analog (carbovir: NSC 614846) against human immunodeficiency virus in vitro. Biochemical and biophysical research communications. 1988 Oct 31;156(2):1046-53.

10. FDA. HIV/AIDS History of Approvals - HIV/AIDS Historical Time Line. 1981-1990. Available at: https://www.fda.gov/forpatients/illness/hivaids/histor y/ucm151074.htm. Accessed May 1, 2017.

11. Horwitz JP, Chua J, Noel M. Nucleosides. V. The Monomesylates of 1-(2'-Deoxy- $\beta$-D-lyxofuranosyl) thymine 1,2. J Org Chem. 1964;29(7):2076-8.

12. Akanbi MO, Scarci K, Taiwo B, Murphy RL. Combination Nucleoside/ Nucleotide Reverse Transcriptase Inhibitors. Expert Opin Pharmacother. 2012;13(1):65.

13. Richman DD, Fischl MA, Grieco MH. The Toxicity of Azidothymidine (AZT) in the Treatment of Patients with AIDS and AIDS-Related Complex. N Engl J Med. 1987;317(4):192-7.

14. Wickramasinghe SN. Diagnosis of megaloblastic anaemias. Blood Rev. 2006;20(6):299-318.

15. Levine AM, Berhane K, Masri-Lavine L, Sanchez M, Young M, Augenbraun M, et al. Prevalence and correlates of anemia in a large cohort of HIV-infected women: Women's Interagency HIV Study. Journal of acquired immune deficiency syndromes (1999). 2001 Jan;26(1):28-35.

16. Ukoha U, Umeasalugo K, Dimkpa U, Ndukwe G, Anyabolu A, Emefo L. Effects of zidovudine on hemostatic and hematologic parameters in adult rats. $\mathrm{J}$ Exp Integr Med. 2015;5(2):85.

17. Raghu R, Jesudas B, Bhavani G, Ezhilarasan D, Karthikeyan S. Silibinin mitigates zidovudine-induced hepatocellular degenerative changes, oxidative stress and hyperlipidaemia in rats. Hum Exp Toxicol. 2015;34(11):1031-42.

18. Okwa IB, Akindele AJ, Agbaje EO, Oshinuga OT, Anunobi CC, Adeyemi OO. Effect of subclinical, clinical and supraclinical doses of calcium channel blockers on models of drug-induced hepatotoxicity in rats. EXCLI J. 2013;12:231-50.

19. Agarwal D, Chakravarty J, Chaube L, Rai M, Agrawal NR, Sundar S. High incidence of zidovudine induced anaemia in HIV infected patients in eastern India. 2010. Available at: http://imsear.li.mahidol.ac.th/handle/123456789/1355 98. Accessed March 21, 2017.

20. Langtry HD, Campoli-Richards DM. Zidovudine. A review of its pharmacodynamic and pharmacokinetic properties, and therapeutic efficacy. Drugs. 1989;37(4):408-50.

21. Lutton JD, Abraham NG, Drummond GS, Levere RD, Kappas A. Zinc porphyrins: Potent inhibitors of hematopoieses in animal and human bone marrow. Proc Natl Acad Sci U S A. 1997;94(4):1432-6.

22. Adewale AO, Jubril OO, Oluwaseun HI, Selimot HA, Ayoola AR. Effects of a single pill 3-drug combination of lamivudine, nevirapine and zidovudine on blood parameters and liver histology in female wistar rats. American Journal of Medicine and Medical Sciences. 2012;2(4):71-4.

23. Gallicchio VS, Hughes NK, Tse KF. Prevention of hematopoietic myeloid and megakaryocyte toxicity associated with zidovudine in vivo in mice with recombinant GM-CSF. Growth Regul. 1994;4(2):417. 
24. Gallicchio VS, Hughes NK, Tse KF, Gaines H. Prevention of the hematopoietic toxicity associated with zidovudine in vivo with. Growth Factors Chur Switz. 1993;9(3):177-83.

25. Shaunak S, Bartlett J. Zidovudine-induced neutropenia: are we too cautious? The Lancet. 1989;334(8654):91-2.

26. Awodele O, Agbaje EO, Adesina EA, Akintonwa A. Hepatoprotective role of neutrosec $\mathrm{R}$ on hepatic damage induced by combination of zidovudine and combined anti-tuberculous agents in rats. Tokai J Exp Clin Med. 2011;36(2):31-6.

27. Sallie R, Michael Tredger J, Williams R. Drugs and the liver part 1: Testing liver function. Biopharm Drug Dispos. 1991;12(4):251-9.
28. Jones AL. Hepatology. A Text Book of Liver Disease. In: Hepatology, Z.D. and Boyer, T.D., Eds. Philadelphia: W.B. Saunders; 1982.

29. Sharma PO. Clinical Biochemistry of Hepatotoxicity. J Clin Toxicol. 2014;4(1).

30. Mehana EE, Meki ARMA, Fazili KM. Ameliorated effects of green tea extract on lead induced liver toxicity in rats. Exp Toxicol Pathol Off J Ges Toxikol Pathol. 2012;64(4):291-5.

Cite this article as: Pandey $\mathrm{R}$, Singh $\mathrm{R}$.

Azidothymidine induces severe hematological toxicity and hepatic injury in Charles Foster rats. Int J Basic Clin Pharmacol 2017;6:2125-30. 